\title{
Modeling and Simulation of Closed-Loop Mechanical Systems for Underwater Applications
}

\author{
Norimitsu Sakagami \\ Department of Navigation and Ocean Engineering \\ Tokai University \\ Shizuoka, Japan 424-0902 \\ Email: sakagami@scc.u-tokai.ac.jp
}

\author{
Katsuya Otomasu \\ Department of Robotics \\ Ritsumeikan University \\ Kusatsu, Shiga, Japan, 525-8577
}

\author{
Song K. Choi \\ Department of Mechanical Engineering \\ University of Hawaii at Manoa \\ Honolulu, HI, USA 96822 \\ Email: schoi@hawaii.edu
}

\begin{abstract}
As described in this paper, we derive the dynamics of closed-loop mechanical systems for underwater applications and present a numerical simulation of an underwater system. The dynamics model derived here is useful for analysis, control, and optimization for underwater parallel mechanisms and underwater object manipulation by multiple arms or fingers. As a first step, a two-dimensional underwater manipulator system with a stationary vehicle is modeled in this work. The dynamics model is coupled with hydrodynamic effects. Using the derived model, we conduct underwater object manipulation based on a force control method for use with underwater manipulators to prevent their contact points from slipping on an object surface. Moreover, we discuss the usefulness of the model and the dynamics of a freefloating vehicle-manipulator system with a closed-loop structure.
\end{abstract}

\section{INTRODUCTION}

Autonomous manipulation for underwater vehicles is a challenging topic in the field of Ocean Engineering. Understanding the related dynamics is important to achieve autonomous manipulation. The dynamics encompasses important information for analysis, control, and optimization of the motion of underwater vehicles and their manipulators. However, the dynamic equations of motion are generally more complicated than those of robots in air. They include added inertia, drag force, and buoyancy force terms as well as inertia, centrifugal and Coriolis forces, along with gravity force terms. Additionally, the vehicle motion is affected by the motion of their manipulators because they are free-floating systems (nonholonomic systems).

Several researchers have derived the dynamics of underwater vehicle-manipulator systems (UVMSs)[1][2][3]. Based on the dynamics, control theories, motion planning, and optimization methods of UVMSs have been discussed and developed. For example, adaptive control[4][5], neural network[6], fuzzy logic[7] have been proposed for UVMSs. One author has developed an UVMS, called a Semi-Autonomous Vehicle for Intervention Missions (SAUVIM), and has conducted sea trials to demonstrate its control performance[8].

The research efforts described above specifically examine the dynamics and control of serial link manipulators for underwater vehicles. They have not discussed closed-loop mechanical systems such as parallel link manipulators. The dynamics of the closed-loop systems are expected to be useful for analysis and control of underwater robotic manipulation and grasping, and underwater humanoid robots, as portrayed in Figure 1. However, the various aspects of the dynamics cannot be derived as easily as those of serial link manipulators. To derive the model of closed-loop systems, one must consider the closed kinematic constraints[9][10]. Once the dynamics model is obtained, it is expected to be useful to perform complex underwater tasks using dual arm systems, and to design new underwater mechanical systems. One author has developed an underwater robot with a dual arm[11] and a parallel mechanism system[12] used to change the pitch angle of an underwater robot for reducing the hydrodynamic force acting on it. The dynamics with the closed-loop constraints is also available for analysis and control of these robotic systems. As described in this paper, we derive the dynamics model with closed kinematic loop constraints for underwater applications. Moreover, we conduct a numerical simulation of underwater object manipulation based on a force control method and friction force between the contact points and an object without slippage on its surface. Analysis of object slipping conditions for contact points is an interesting research topic. The conditions are dependent on the hydrodynamic forces acting on the links and the object, and the friction coefficients between the contact points and the object.

The contributions of this paper are two-fold: First, we discuss the derivation of the dynamics model of an underwater manipulator system with a closed kinematic constraint. Consequently, we can use the model for underwater robotic manipulation and grasping, and use the model for designing new underwater mechanical systems. The dynamics model is applicable to analyze and control the motion of underwater closed-loop mechanical systems. Second, we propose an object manipulation method for use with underwater manipulators to prevent their contact points from slipping on an object surface in the event of a disturbance.

This paper presents the following. In Section 2, we describe related works on dynamics of UVMSs and parallel mechanisms and our work. Section 3 discusses the derivation of the dynamics model of an underwater manipulator system with a closed kinematic loop constraint. In Section 5, we present a graphical simulator using OpenGL library and present a numerical result to assess the object manipulation performance. 


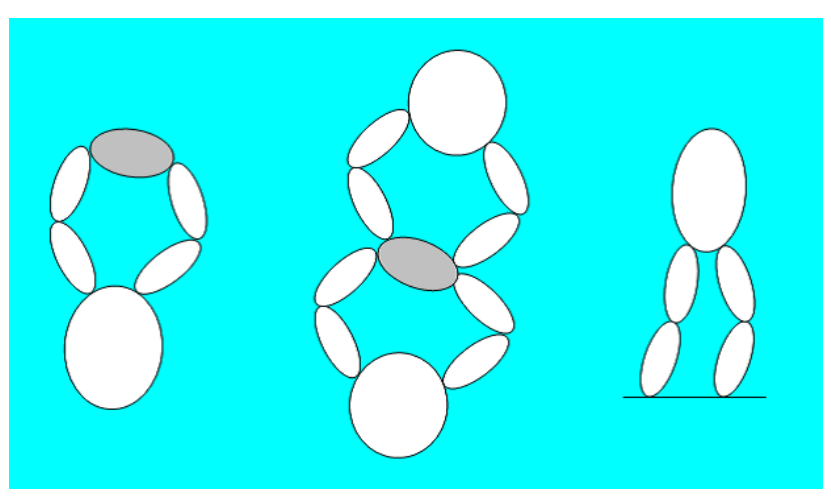

Fig. 1. Closed kinematic loop systems.

\section{RELATED WORK}

The dynamics of ocean vehicles has been summarized systematically and has been discussed in the literature[13]. Ocean vehicles are free-floating systems called non-holonomic systems according to their physical properties. Added inertia, hydrodynamic damping, and restoring forces act on ocean vehicles. Added inertia force results from the inertia of surrounding fluid of the vehicles. Hydrodynamic damping is caused by vortex shedding, skin friction, and other phenomena. Restoring force results from Archimedes' principle. Based on the dynamics, control and stability theory have also been discussed in [13].

UVMSs are also non-holonomic systems and their equations of motion also include the added inertia, hydrodynamic damping, and restoring force terms. Several reports in the literature[1][2] have presented derivation of the dynamics of UVMSs. In one study[1], the dynamics of underwater vehicles with a manipulator system is derived, and hybrid position/force control is simulated numerically. The paper[2] derives the dynamics of UVMSs, with discussion of the physical properties of the dynamics. The control design is also discussed in terms of feedback linearization. The dynamics and control theory have been summarized systematically in [3].

As evidenced by the reports explained above, control theories and motion planning algorithms have been discussed and developed extensively up to the present day. Some researchers have conducted field experiments with UVMSs to demonstrate their control performance [8][14][15]. One author developed a UVMS called SAUVIM and conducted sea trials with it[8]. This report is the first of a successful sea trial outcome among UVMSs worldwide.

Previous studies have specifically examined the modeling and control of serial link manipulators for underwater vehicles, which comprise serial kinematic chains formed by rigid bodies and joints. Other types of manipulators are used in the fields of robotics and mechanical engineering. One type is parallel link mechanisms with closed kinematic chains. Parallel link mechanisms are widely used for high-speed, lightweight, and high-precision robots because their actuators can be located on the base of the robots. Derivation of the dynamics is more complicated than that of the dynamics of serial link manipulators because of the closed kinematic loop constraints. Although the dynamics with the constraints cannot be obtained easily, it is available to control, analyze, and optimize the motion of the mechanical systems such as parallel link manipulators, robotic manipulation and grasping systems, and so on (Figure 1).

Three main approaches are used to derive the dynamics: Newton-Euler, Direct Lagrangian, and Lagrange-D'Alembert formulation on the reduced system[10]. Using the third method, one or more joints are cut to form a tree system[9] (or a reduced system). Then we obtain the equations of motion using the loop constraints and the D'Alembert principle. Using the method, however, we cannot consider external torque for the cut joint(s). Using another method[10], all the joint torque can be included in the equations of motion by cutting one or more links. All the external forces and torque acting on links can be considered.

As described in this paper, we derive the dynamics of a closed-loop system (a manipulator system) for underwater applications using a method described in an earlier report[10]. Here, we assume that the manipulator system is fixed with a stationary vehicle, which is a holonomic system (not a free-floating system). The dynamics derived here for analysis, control, and optimization of underwater robotic systems includes hydrodynamic terms. Using the derived model, we conduct underwater object manipulation based on a force control method.

\section{Modeling}

This section explains the derivation of an underwater manipulator system with a closed-loop constraint. First, we derive the dynamics equations of motion of the rigid bodies. Then we add the hydrodynamic terms to the dynamics.

\section{A. Dynamics on rigid bodies}

We derive the dynamic equations of motion of rigid bodies with a closed-loop constraint. The derivation in this subsection is based on that presented in an earlier report [10].

Assuming that two manipulators are fixed on a stationary vehicle and that their motion is in two dimensions, as shown in Figure 2, the manipulator system and the object constitute a closed-loop structure.

First, we cut a link of the closed-loop structure as shown in Figure 2 and form a tree system (two serial manipulators). Then, the dynamics of the manipulators without the hydrodynamic terms are obtained easily as

$$
\begin{gathered}
M_{1} \ddot{\boldsymbol{\theta}}_{1}+\boldsymbol{C}_{1}=\boldsymbol{\tau}_{1} \\
\boldsymbol{M}_{2} \ddot{\boldsymbol{\theta}}_{2}+\boldsymbol{C}_{2}=\boldsymbol{\tau}_{2},
\end{gathered}
$$

where $\boldsymbol{M}_{i}$ represents the inertia matrix of the $i$-th serial link manipulator and $C_{i}$ is the centrifugal and Coriolis term. The vectors shown as $\boldsymbol{\tau}_{i}=\left(\tau_{i 1}, \tau_{i 2}, \tau_{i 3}\right)^{T}$ and $\boldsymbol{\theta}_{i}=\left(\theta_{i 1}, \theta_{i 2}, \theta_{i 3}\right)^{T}$ respectively denote the joint torque vector and the joint angle 

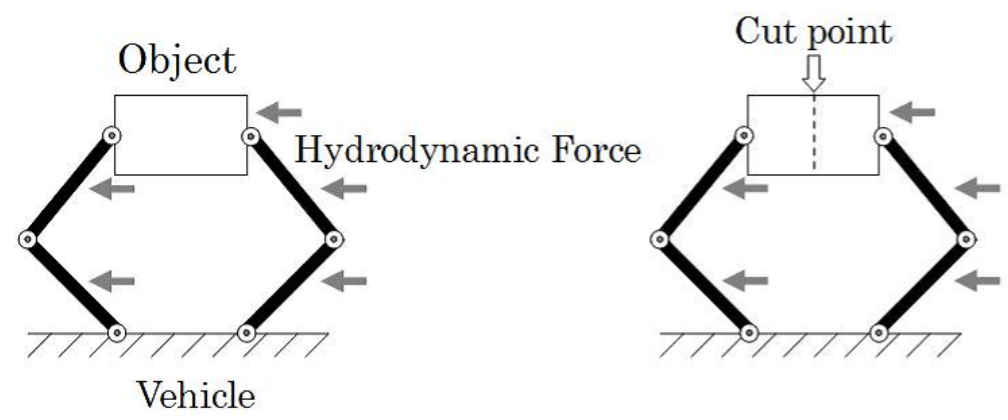

1st manipulator 2nd manipulator

Fig. 2. Modeling an underwater manipulator system and an object.

vector of the $i$-th manipulator. Consequently, we obtain the dynamics of the closed-loop mechanical system

$$
\boldsymbol{\Phi}^{T}\left[\begin{array}{c}
\boldsymbol{M}_{1} \ddot{\boldsymbol{\theta}}_{1}+\boldsymbol{C}_{1} \\
\boldsymbol{M}_{2} \ddot{\boldsymbol{\theta}}_{2}+\boldsymbol{C}_{2}
\end{array}\right]=\boldsymbol{\Phi}^{T}\left[\begin{array}{c}
\boldsymbol{\tau}_{1} \\
\boldsymbol{\tau}_{2}
\end{array}\right]
$$

using a matrix $\boldsymbol{\Phi}^{T}$ composed of the Jacobian matrices of the constraint equation (closed kinematics)[10]. Therein, the righthand term is the generalized torque $\tau_{c}$ for the closed-loop system.

\section{B. Hydrodynamic terms}

Next, we obtain the hydrodynamic terms for the equations of motion of the manipulator system. When forces are applied to their links for closed-loop mechanisms, the generalized torque $\tau_{c}$ can be given as shown below.

$$
\boldsymbol{\tau}_{c}=\boldsymbol{\Phi}^{T}\left[\begin{array}{c}
\boldsymbol{\tau}_{1}+\sum_{i} \boldsymbol{J}_{1 i}^{T}\left(\boldsymbol{\theta}_{1}\right) \boldsymbol{f}_{1 i} \\
\boldsymbol{\tau}_{2}+\sum_{j} \boldsymbol{J}_{2 j}^{T}\left(\boldsymbol{\theta}_{2}\right) \boldsymbol{f}_{2 j}
\end{array}\right]
$$

Therein, $\boldsymbol{J}$ is the Jacobian matrix of a point of application of hydrodynamic forces $\boldsymbol{f}_{1 i}$ and $\boldsymbol{f}_{2 j}$. The external forces $\boldsymbol{f}_{1 i}$ and $\boldsymbol{f}_{2 j}$ can be incorporated into the generalized force space using Eq. (4).

Consequently, the manipulator system dynamics is expressed as shown below.

$$
\boldsymbol{\Phi}^{T}\left[\begin{array}{c}
\boldsymbol{M}_{1} \ddot{\boldsymbol{\theta}}_{1}+\boldsymbol{C}_{1} \\
\boldsymbol{M}_{2} \ddot{\boldsymbol{\theta}}_{2}+\boldsymbol{C}_{2}
\end{array}\right]=\boldsymbol{\Phi}^{T}\left[\begin{array}{c}
\boldsymbol{\tau}_{1}+\sum_{i} \boldsymbol{J}_{1 i}^{T}\left(\boldsymbol{\theta}_{1}\right) \boldsymbol{f}_{1 i} \\
\boldsymbol{\tau}_{2}+\sum_{j} \boldsymbol{J}_{2 j}^{T}\left(\boldsymbol{\theta}_{2}\right) \boldsymbol{f}_{2 j}
\end{array}\right]
$$

To model the hydrodynamic forces, we assume that the added mass and damping forces act as external forces on a strip of the links and the object[16]. These forces acting on a strip are modeled as follows.

$$
\boldsymbol{f}_{1 i}=C_{m} \rho \frac{\pi D_{i}^{2}}{4} d l \dot{\boldsymbol{v}}_{i}+\frac{1}{2} C_{d} \rho D_{i} d l\left|\boldsymbol{v}_{i}\right| \boldsymbol{v}_{i}
$$

In that equation, $C_{m}$ and $C_{d}$ respectively stand for the added mass and drag coefficients, $\rho$ represents the fluid density, $D_{i}$ signifies the diameters of the links or the object, $d l$ denotes the strip length. The force $f_{2 i}$ in Eq. (5) has also the same form as Eq. (6). The drag coefficient $C_{d}$ is normally dependent on the angle of attack $\alpha$. However, in this paper, we treat the drag coefficient as constant to simplify the model. We define the relative flow velocity $\boldsymbol{v}_{i}$ of the links or the object to the flow velocity $\boldsymbol{v}_{f}$ as shown below.

$$
\boldsymbol{v}_{i}=\boldsymbol{v}_{l i}-\boldsymbol{v}_{f}
$$

Therein, $\boldsymbol{v}_{l i}$ is the velocity of a strip of the link or object.

In the model of the hydrodynamic force (6), we neglect the lift force which is the force perpendicular to the flow velocity because we assume that the motion of the manipulator system and the flow velocity are in two dimensions for these analyses.

For numerical simulation, we consider the following timevariant flow velocity.

$$
\boldsymbol{v}_{f}=\left(v_{x}(t), v_{y}(t)\right)^{T}
$$

The magnitude and direction of $v_{x}(t)$ and $v_{y}(t)$ change irregularly with time. Using the model described above, we conduct a numerical simulation of the manipulator system, as described in the next section.

\section{Numerical Simulation}

This section presents a two-dimensional numerical analysis undertaken to assess the performance of the object manipulation based on a force control method and the friction force between the end-effectors and an object under external disturbances.

\section{A. Manipulation Simulator}

We have developed the manipulation simulator shown in Figure 3 for underwater manipulation under external disturbances. The code was written in $\mathrm{C}$ language using Visual Studio 2010 (Microsoft Corp.). The graphical interface was developed using OpenGL library. The derived dynamics was solved numerically using the Runge-Kutta-Gill method in the simulation. The time step size was set to 0.001 [s]. Physical parameters of the manipulator system and object can be adjusted arbitrarily along with the external disturbance.

We assume that angle sensors in the manipulator joints measure the joint angle, and that force sensors attached to the end-effectors measure the force from the object. Force noise $\Delta F$ generated using the Mersenne Twister algorithm[17] was artificially added to the force signals from the force sensors in the simulation. The range of noise amplitude was -1.0 and 

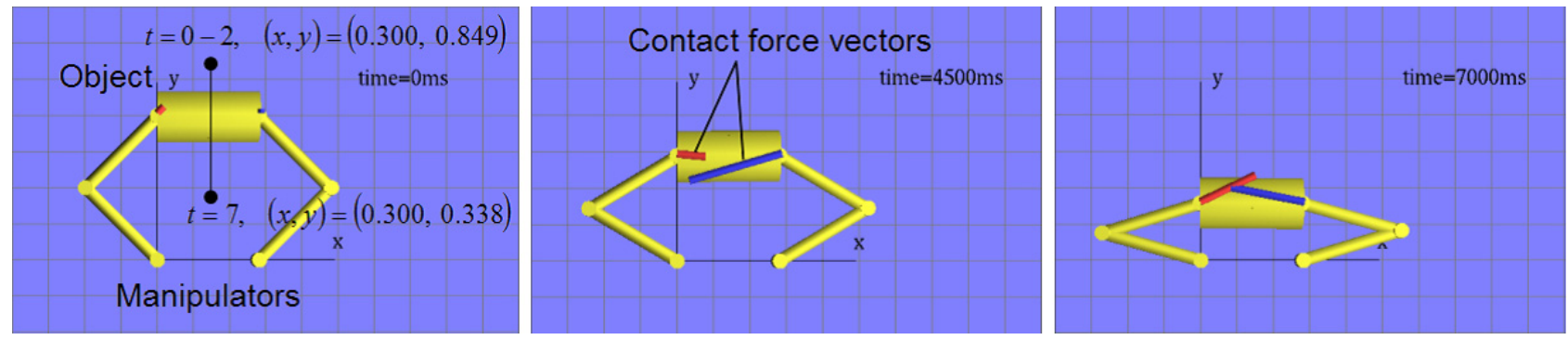

Fig. 3. Graphical simulator developed to examine the manipulator system motion. The two 2-DOF manipulators handle the object underwater.

TABLE I

PARAMETERS USED FOR SIMULATION.

\begin{tabular}{|c||c|}
\hline Added mass coefficients $C_{m}$ & 1.0 \\
Drag coefficients $C_{d}$ & 1.2 \\
Link diameter $D_{i}$ & $0.05[\mathrm{~m}]$ \\
Object diameter $D$ & $0.15[\mathrm{~m}]$ \\
Link \& Object length & $0.6[\mathrm{~m}]$ \\
Link \& Object weight & $1.0[\mathrm{~kg}]$ \\
Link \& Object tensor & $0.001\left[\mathrm{kgm}{ }^{2}\right]$ \\
Strip length $d l$ & $0.1[\mathrm{~m}]$ \\
Fluid density $\rho$ & $1000\left[\mathrm{~kg} / \mathrm{m}^{3}\right]$ \\
\hline
\end{tabular}

TABLE II

PARAMETERS OF IRREGULAR WAVES.

\begin{tabular}{|c||ccccc|}
\hline & $k=1$ & $k=2$ & $k=3$ & $k=4$ & $k=5$ \\
\hline$a_{k}$ & 0.02 & 0.05 & 0.07 & 0.09 & 0.10 \\
$T_{k}$ & 3.76 & 5.04 & 6.63 & 8.69 & 13.03 \\
$\phi_{k}$ & $-\pi / 2$ & $\pi / 3$ & $-\pi$ & $\pi / 6$ & $2 \pi / 3$ \\
\hline
\end{tabular}

$1.0[\mathrm{~N}]$. The force signals were filtered with a Kalman filter for force control.

\section{B. Underwater manipulation}

We conducted a numerical simulation of underwater manipulation incorporating the friction force between the contact points and the object without slipping on its surface.

For this simulation, the manipulator system and the object were two-dimensional. Each manipulator had two rotational joints. The first joint was fixed with the base. Their endeffectors contacted with the object at two points. The manipulators brought the object closer to the base, as portrayed in Figure 3 . The contact type is classified into point contact with friction[18]. The physical parameters of the system and object used in the simulation are presented in Table I. The gravity force was not included in this model. However, it is easy for us to derive the gravity term using the method explained above.

We assumed that the manipulators moved the object from the starting position $(x, y)^{T}=(0.300,0.849)^{T}$ to the end po- sition $(0.300,0.338)^{T}$ as shown in Figure 3. During the first 2 seconds, the desired position of the object was $(0.300,0.849)^{T}$ to ensure that the manipulators generated sufficient contact force. The desired orientation of the object was set as 0 [deg] during the movement.

We used a conventional Proportional Derivative (PD) feedback controller with high gain to increase the stiffness and damping of the 2-DOF left manipulator.

$$
\boldsymbol{\tau}_{1}^{\prime}=\boldsymbol{J}_{1}^{\prime T}\left[\boldsymbol{K}_{p}\left(\boldsymbol{x}_{d 1}-\boldsymbol{x}_{1}\right)-\boldsymbol{K}_{d} \dot{\boldsymbol{x}}_{1}\right]
$$

In this expression, $\tau_{1}^{\prime}$ is the joint torque vector for the left manipulator, $\boldsymbol{J}_{1}^{\prime T}$ stands for the Jacobian matrix, $\boldsymbol{K}_{p}$ and $\boldsymbol{K}_{d}$ are the diagonal feedback gain matrices. The diagonal component of $\boldsymbol{K}_{p}$ is 1500 . That of $\boldsymbol{K}_{d}$ is 1.5. $\boldsymbol{x}_{d 1}$ and $\boldsymbol{x}_{1}$ are the desired position and actual position of the end-effector (contact point).

We used a force feedback controller for the 2-DOF right manipulator.

$$
\boldsymbol{\tau}_{2}^{\prime}=\boldsymbol{J}_{2}^{\prime T}\left[\begin{array}{cc}
c_{\theta} & -s_{\theta} \\
s_{\theta} & c_{\theta}
\end{array}\right]\left[\begin{array}{c}
K_{f} \int\left(f_{x^{\prime} d}-f_{x^{\prime} 2}\right) \mathrm{d} t \\
K_{p}\left(y_{d}^{\prime}-y^{\prime}\right)-K_{d} \dot{y}^{\prime}
\end{array}\right]
$$

Therein, $\tau_{2}^{\prime}$ stands for the joint torque vector for the right manipulator, $\boldsymbol{J}_{2}^{\prime} T$ represents the Jacobian matrix, $c_{\theta}=\cos \theta$, $s_{\theta}=\sin \theta, K_{f}(=2.5)$ signifies the force feedback gain, $K_{p}(=$ $1500)$ and $K_{d}(=1.5)$ denote the PD feedback gains, $f_{x^{\prime} d}$ represents the desired force to prevent their end-effectors from slipping on the object surface, and $f_{x^{\prime} 2}$ is the force signal from the force sensor mounted on the right manipulator.

The desired force $f_{x^{\prime} d}$ was designed to ensure that the manipulators handled the object without slipping on its surface. Using friction forces $f_{y^{\prime} 1}$ and $f_{y^{\prime} 2}$ in Figure 4 from the force sensors and the friction coefficient, the desired force was set as follows:

$$
f_{x^{\prime} d}= \begin{cases}k\left|f_{y^{\prime} 2}\right| / \mu+k\left|f_{y^{\prime} 1}\right| & / \mu-f_{x^{\prime} 1} \\ & \left(k\left|f_{y^{\prime} 1}\right| / \mu \geq f_{x^{\prime} 1}\right) \\ k\left|f_{y^{\prime} 2}\right| / \mu & \left(k\left|f_{y^{\prime} 1}\right| / \mu<f_{x^{\prime} 1}\right)\end{cases}
$$

when $\left|f_{y^{\prime} 1}\right| \leq\left|f_{y^{\prime} 2}\right|$. 


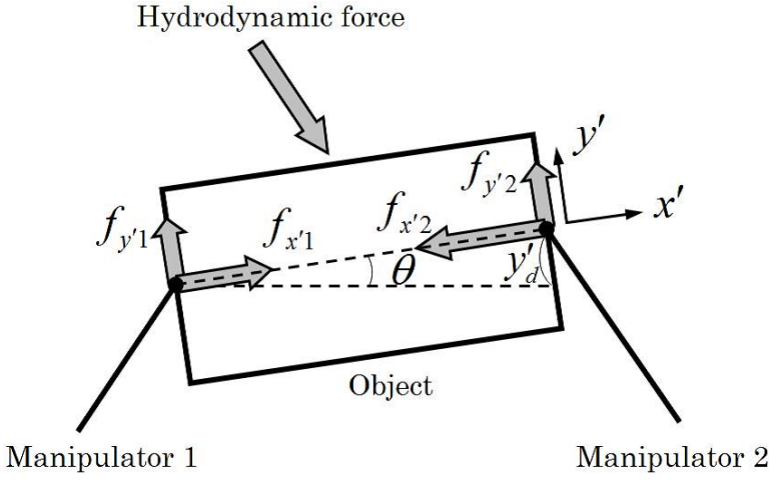

Fig. 4. Force acting on the object.

On the other hand, when $\left|f_{y^{\prime} 1}\right|>\left|f_{y^{\prime} 2}\right|$,

$$
f_{x^{\prime} d}= \begin{cases}k\left|f_{y^{\prime} 1}\right| / \mu-f_{x^{\prime} 1}+ & k\left|f_{y^{\prime}}\right| / \mu \\ k\left|f_{y^{\prime} 1}\right| / \mu & \left(k\left|f_{y^{\prime} 2}\right| / \mu \geq f_{x^{\prime} 2}\right) \\ & \left(k\left|f_{y^{\prime} 2}\right| / \mu<f_{x^{\prime} 2}\right)\end{cases}
$$

where $k$ signifies the safety coefficient of 2.0 , and $\mu(=1.0)$ is the friction coefficient obtained using the underwater experimentally obtained results[19]. The minimum value for $k\left|f_{y^{\prime} 1}\right| / \mu$ and $k\left|f_{y^{\prime} 2}\right| / \mu$ was set as $3.0[\mathrm{~N}]$.

We investigated the object manipulation performance with time-variant flow velocity.

$$
v_{x}(t)=v_{y}(t)=\sum_{k=1}^{5} a_{k} \cos \left(2 \pi \frac{t}{T_{k}}+\phi_{k}\right)
$$

Five velocities were superposed to produce an irregular flow velocity. The parameters $a_{k}, T_{k}$, and $\phi_{k}$ respectively represent the amplitude, frequency, and phase lag of the five flow velocities. The values of these parameters are presented in Table II.

The results of the object trajectories in the flow velocity are shown in Figure 5. Under the disturbance, the positional error was sufficiently small after 2 seconds (after the manipulators generated sufficient contact force). The force patterns are presented in Figures 6 and 7. The generated force was sufficient for the manipulators to handle the object without slipping on its surface during the motion (after 2 seconds). The contact force acting on the right manipulator was larger than that acting on the left manipulator because of the flow velocity (Eq.(13))

\section{Discussion}

One important contribution of this research is the dynamics model, which is useful for the design of underwater parallel mechanisms. Parallel mechanisms are widely used for highspeed, lightweight, and high-precision robots because their actuators can be located on the base of the robots. The derived model will be considerably important for the design, control, and optimization of the underwater parallel mechanisms. To reduce the hydrodynamic effects, the simulator is useful to

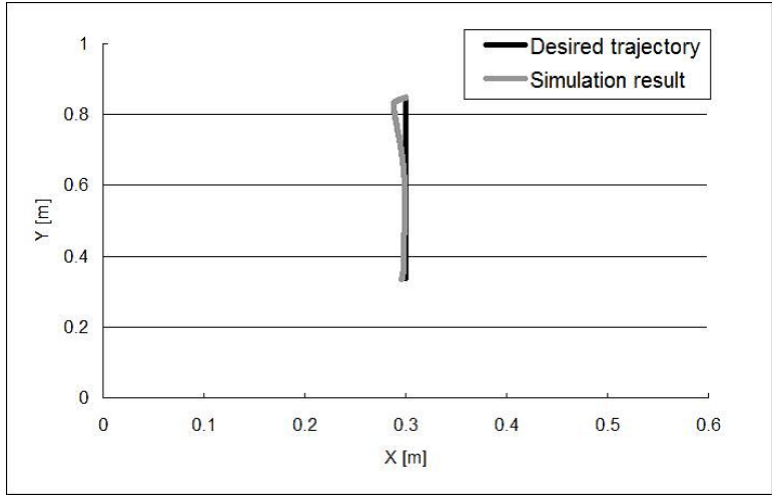

Fig. 5. Object trajectory during manipulation.

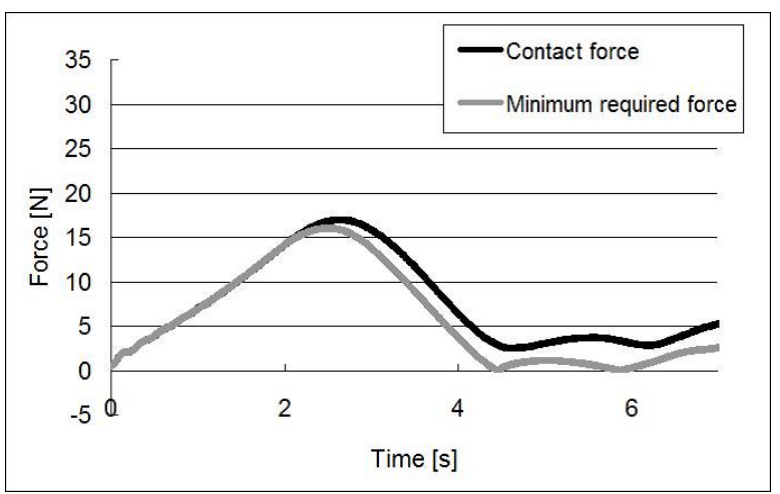

Fig. 6. Contact force $f_{x^{\prime} 1}$ obtained when handling the object without slipping (left contact point). The minimum required force was estimated from the friction force $f_{y^{\prime} 1}$ and the friction coefficient.

investigate the torque patterns by adjusting the physical parameters.

The next steps of this research are to increase the DOF of the manipulators to achieve complex tasks, develop a threedimensional simulator, and to consider a free-floating UVMS. In this work, we assumed that the underwater manipulators were fixed with the stationary vehicle, without a free-floating vehicle. These manipulators belong to holonomic systems from physical properties. Therefore, we were able to derive the dynamics with the holonomic constraint using the method[10]. However, the method used here cannot be applied in the case of dynamics with non-holonomic constraints. To derive the dynamics with non-holonomic constraints, such as the case of a free-floating vehicle-manipulator system as presented in Figure 8, a different approach must be applied. Derivation of the dynamics of closed-loop mechanical systems with nonholonomic constraints is the primary goal of our research under disturbances.

\section{CONCLUSION}

In this work, we derived the dynamics of a dual underwater manipulator system and discussed underwater object manipulation based on a force control method and the friction 


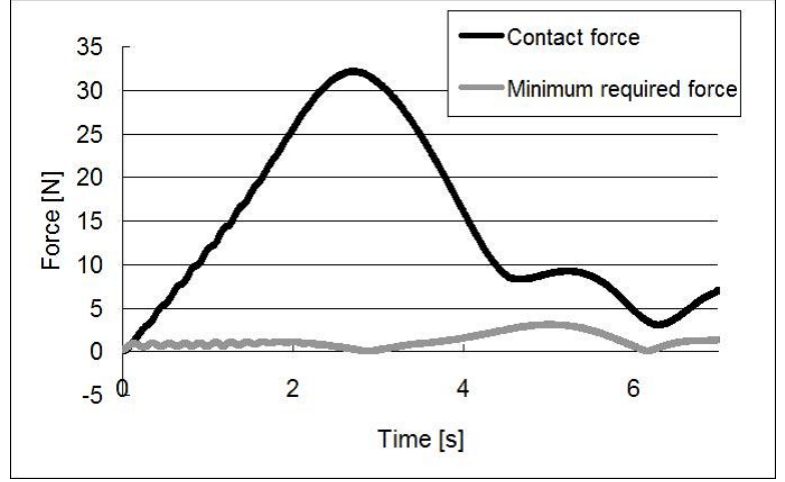

Fig. 7. Contact force $f_{x^{\prime} 2}$ to handle the object without slipping (right contact point). The minimum required force was estimated from the friction force $f_{y^{\prime} 2}$ and the friction coefficient.

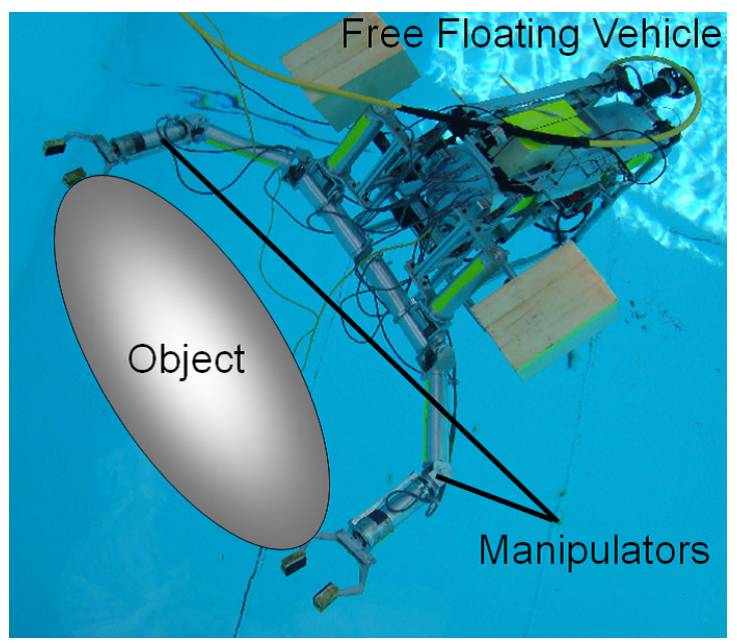

Fig. 8. Dual manipulator system.[11]

force between the contact points and the object. Although we assumed the manipulator system as fixed in a stationary basement, the dynamics was coupled with hydrodynamic effects. The derived dynamics with a closed-loop mechanical structure is useful for analysis, control, and optimization for underwater applications, such as design of parallel mechanisms and control of underwater object manipulation by multiple arms or fingers. We also presented the numerical simulation of the underwater manipulation As a subject of future work, we plan to derive the dynamics of a free-floating system using a closedloop structure.

\section{ACKNOWLEDGMENT}

The authors would like to thank Tokai University which provided financial support (overseas research visit program) for the research activity.

\section{REFERENCES}

[1] K. Ioi, and K. Itoh, Modeling and Simulation of an Underwater Manipulator, Advanced Robotics, Vol.4, No.4, pp.303-317, 1989.
[2] I. Schjolberg,. and T. I. Fossen, Modelling and Control of Underwater Vehicle-Manipulator Systems, John Wiley \& Sons Ltd., 1994.

[3] G. Antonelli, Underwater Robots: Motion and Force Control of VehicleManipulator Systems, Springer Berlin Heidelberg, 2006.

[4] H. Mahesh, J. Yuh and R. Lakshmi, Control of underwater robots in working mode, Proceedings of the 1991 IEEE Int. Conf. on Robotics and Automation, pp.2630-2635, Sacramento CA, 1991.

[5] G. Antonelli and E. Cataldi, Recursive adaptive control for an underwater vehicle carrying a manipulator, Proceedings of 22nd Mediterranean Conference on Control and Automation (MED), pp.847-852, Palermo, Italy, 2014.

[6] C. H. Santos, R. Guenther and E. D. Pieri, A reactive neural network architecture to redundancy resolution for underwater vehicle manipulator systems, Proceedings of the 2006 IEEE Int. Conf. on Robotics and Automation, pp.3238-3243, Orlando, FL, 2006.

[7] Y. Cui and N. Sarkar, A unified force control approach to autonomous underwater manipulation, Proceedings of the 2000 IEEE Int. Conf. on Robotics and Automation, pp.1263-1268, San Francisco, CA, 2000.

[8] G. Marani, S. K. Choi, and J. Yuh, Underwater autonomous manipulation for intervention missions AUVs, Journal of Ocean Engineering, Vol.36, No.1, pp.15-23, 2009.

[9] Y. Nakamura and M. Ghodoussi, A computational scheme of closed link robot dynamics derived by D'Alembert principle, Proceedings of the 1988 IEEE International Conference on Robotics and Automation, pp.13541360, 1988.

[10] Y. K. Yiu, H. Cheng, Z. H. Xiong, G. F. Liu, and Z. X. Li, On the Dynamics of Parallel Manipulators, Proceedings of the 2001 IEEE International Conference on Robotics and Automation, pp.3766-3771, 2001.

[11] N. Sakagami, M. Shibata, S. Kawamura, T. Inoue, H. Onishi, S. Murakami, An Attitude Control System for Underwater Vehicle-Manipulator Systems, Proceedings of IEEE International Conference on Robotics and Automation (ICRA), pp.1761-1767, 2010.

[12] K. Yokoi, M. Kawabata, S. Sakai, S. Kawamura, N. Sakagami, S. Matsuda, A. Mitsui, K. Sano, Improvement of a Human-portable Underwater Robot for Soil Core Sampling, Proceedings of OCEANS MTS/IEEE 2014 140326-187, 2014.

[13] T. I. Fossen, Guidance and Control of Ocean Vehicles, Proceedings of the Third Conference on Marine Craft Maneuvering and Control, pp.45 57, 1994.

[14] P. Ridao, M. Carreras, D. Ribas, P. J. Sanz, and G. Oliver, Intervention AUVs: the next challenge, Proceeding of the IFAC World Congress, Vol 19, part 1, pp. 12146-12159, 2014

[15] J. Evans, P. Redmond, C. Plakas, K. Hamilton and D. Lane, Autonomous docking for Intervention-AUVs using sonar and video-based real-time $3 D$ pose estimation, Proceedings of OCEANS 2003, pp. 2201-2210, 2003.

[16] T. W. McLain and S. M. Rock, Experiments in the Coordination of Underwater Manipulator and Vehicle Control, Proceedings of OCEANS 1995, San Diego, October, 1995.

[17] M. Matsumoto and T. Nishimura, Mersenne Twister: A 623 dimensionally equidistributed uniform pseudorandom number generator, ACM Transactions on Modeling and Computer Simulation, Vol. 8, No.1, pp.3-30, 1998.

[18] A. M. Okamura, N. Smaby and M. R. Cutkosky, An overview of dexterous manipulation, Proceedings of ICRA 2000, San Francisco, 2000

[19] N. Sakagami, K. Ishimaru, S. Kawamura, M. Shibata, H. Onishi, S. Murakami, "Development of an Underwater Robotic Inspection System using Mechanical Contact", Journal of Field Robotics, Vol.30, Issue 4 pp.624-640, 2013. 